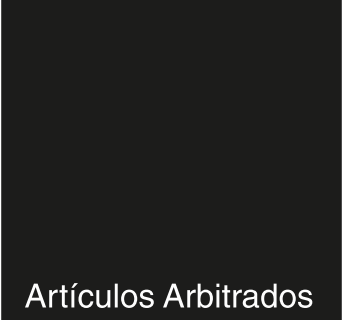

\title{
Espacios verdes en ciudades de zona árida. Diagnóstico de la situación actual de plazas de la ciudad de Mendoza, Argentina
}

\section{Susana Stocco}

Especialista en desarrollo sustentable. Arquitecta, becaria posdoctoral del INAHE, Instituto de Ambiente, Hábitat y Energía; CONICET, Mendoza, Argentina.

sstocco@mendoza-conicet.gob.ar.

\section{María Alicia Cantón}

DAE. Arquitecta, investigadora independiente del INAHE, Instituto de Ambiente, Hábitat y Energía; CONICET, Mendoza, Argentina.

macanton@mendoza-conicet.gob.ar.

\section{Erica Correa}

Doctora en Ciencias, Ing. Química, investigadora independiente del INAHE, Instituto de Ambiente, Hábitat y Energía; CONICET, Mendoza, Argentina. ecorrea@mendoza-conicet.gob.ar.

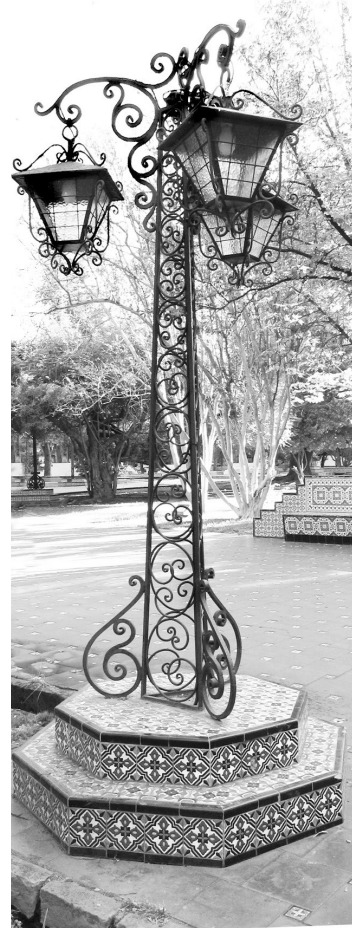




\section{Resumen}

El modelo de desarrollo urbano de la ciudad de Mendoza difiere del adoptado en climas áridos. Su concepción urbanística está basada en la presencia de vegetación a lo largo de calles, plazas, parques y en cada manzana a través de patios. El desarrollo urbano del siglo XX fue debilitando esta trama verde característica; particularmente "las plazas" fueron perdiendo espacio en la trama urbana, y se priorizó la incorporación de materiales sellados ante las superficies verdes, lo que transformó estos espacios en paisajísticamente agradables, pero con un empobrecimiento de las condiciones de habitabilidad de la ciudad. Este trabajo hace un análisis de la configuración morfológica y la materialidad de las plazas atendiendo al carácter relevante de estas en la trama urbana de la ciudad. Busca elaborar un diagnóstico de la situación actual, con el objetivo final de definir pautas de diseño tendientes a maximizar los beneficios ambientales y sociales que sean de utilidad para diseñadores y proyectistas.

Palabras clave

Desarrollo urbano; vegetación; plazas.

\section{Abstract}

Green spaces in cities in arid zones. Diagnosis of the current situation of squares of the city of Mendoza, Argentina.

The urban development model of the city of Mendoza differs from that adopted in arid climates. Its urban design is based on the presence of vegetation along streets, squares, parks and in each block around the courtyards. The urban development of the twentieth century was weakened the characteristic green pattern. Particularly the "squares" were losing space in the urban fabric through prioritizing the incorporation of sealed materials replacing green surfaces, these spaces became pleasant townscapes but impoverished the habitability conditions of the city. This paper analyzes the morphological configuration and the materiality of the squares considering their significance in the urban pattern of the city, seeking to develop a diagnosis of the current situation with the goal of defining design guidelines, tending to maximize environmental and social benefits, that are useful to designers.

\section{Keywords}

Urban development; vegetation; squares. 
Espacios verdes en ciudades de zona árida.

Diagnóstico de la situación actual de plazas de la ciudad de Mendoza, Argentina

\section{Introducción}

En los comienzos de los siglos XVI y XVII, la incorporación de espacios verdes y arboledas en los medios urbanos se inició con el objetivo primario de proveer escenarios adecuados para actividades recreativas y sociales, como un aporte a la belleza del entorno urbano. En el siglo XIX, los efectos de la Revolución Industrial produjeron consecuencias negativas en las urbanizaciones, como el crecimiento excesivo y desordenado de la población, el acrecentamiento de la contaminación, que causaron problemas de hacinamiento, malas condiciones salubridad y el aumento de enfermedades. Una estrategia para revertir los fenómenos descritos y encauzar el desarrollo en el camino de la sustentabilidad es el enverdecimiento urbano. Es decir, incorporar obra viva (árboles, parques y plazas) con el objetivo de generar "Les Conditions de Nature” (RIBAs I PIERA, 2002).

Este concepto tiene como fin priorizar elementos esenciales de la naturaleza dentro de los espacios construidos, como el aire, el agua, el sol y principalmente el uso de la vegetación en los espacios abiertos. En las últimas décadas se ha incrementado progresivamente la conciencia y el conocimiento sobre la importancia que revisten los espacios verdes debido a sus efectos beneficiosos sobre las condiciones ambientales de los medios urbanos. Si bien las áreas verdes no son la solución a los problemas urbanos actuales, contribuyen a resolver y mejorar varios problemas ambientales, sociales e incluso económicos, y propician un ambiente deseable y saludable en el cual vivir (SORENSEN ET ÁL., 1998).

En cuanto a los beneficios ambientales, se pueden diferenciar los efectos que producen una modificación en el microclima, "beneficios climáticos”, y los que generan mejoras en la calidad del aire, "beneficios no climáticos” (López FALFÁN, 2008). Entre los beneficios climáticos, particularmente la vegetación contribuye a disminuir la intensidad de la "isla de calor urbana”, en climas con importantes niveles de radiación solar (CORREA, 2006), favorece la rehidratación de la atmósfera en ciudades de climas áridos, el refrescamiento del aire y la 
consecuente reducción de las cargas térmicas de verano (SANTAMOURIS, 2001 y Mc PHERSON ET ÁL., 1995); en consecuencia, produce mejoras significativas en las condiciones de confort (ACERO ET ÁL., 2010). Entre los efectos no climáticos se destaca el rol de la vegetación como un instrumento de retención temporal de partículas que se encuentran en la atmósfera (SMITH, 1990), que ayuda a disminuir la cantidad de microbios por $\mathrm{m}^{3}$ de aire, actúa como reductor de la contaminación al absorber contaminantes atmosféricos gaseosos (McPHERSON, 1998 y NowAK, 2002). Por otro lado, colabora con la absorción de ruidos mediante el follaje de los árboles y aporta al enriquecimiento de la biodiversidad (SORENSEN ET ÁL., 1998).

Respecto de los beneficios sociales, los espacios verdes son importantes para la salud física y mental, ya que producen estados fisiológicos más distendidos, aminoran la fatiga mental, disminuyen los niveles de estrés y producen bienestar personal, físico y emocional (KaPLAN, 1993; Arddone et ÁL., 1996; Conway, 2000 y Kuo ET ÁL., 1998). Contribuyen al fortalecimiento de las relaciones entre ciudadanos y aumentan la integración e interacción entre los vecinos (Coley et ÁL., 1997, Nikolopoulou ET ÁL., 2003). La vegetación produce efectos positivos en la salud de las personas que utilizan los parques locales con frecuencia (ULRICH, 1984). La presencia de árboles en los espacios urbanos permite bloquear la exposición a los rayos ultravioletas y, en consecuencia, disminuye daños a la salud tales como cáncer de la piel y cataratas (HeISLER ET ÁL., 1994). El incremento de las condiciones de confort en los espacios públicos favorece el uso recreativo y presta un aporte significativo a la estética urbana (McPherson, 1988).

Finalmente, en cuanto a sus beneficios económicos, se observa que en viviendas con una adecuada utilización de la vegetación, el uso de energías disminuye en el orden del 20 o 25 \% (HeIsLer, 1986) y presenta un ahorro del 50 \% al 65 \% (combinando la vegetación urbana con estrategias como cubiertas y fachadas vegetadas) (McPHERson, 1994). Por otra parte, la proximidad a parques públicos o áreas naturales tiene un efecto significativo sobre el valor de las propiedades (Priego GonZalez de CANALEs, 2002; Irarrázaval, 2012). Estos espacios verdes poseen valor estético, histórico y recreativo, lo que permite mostrar a las ciudades más atractivas para los visitantes para promoverlas como destino turístico y generar empleo e ingresos.

Los beneficios descritos guardan estrecha relación con la proporción de espacios abiertos vegetados presentes en las ciudades. Actualmente existen estándares internacionales que 
Espacios verdes en ciudades de zona árida. Diagnóstico de la situación actual de plazas de la ciudad de Mendoza, Argentina

permiten calificar y cuantificar la cantidad de espacios verdes públicos en la trama urbana. A principios de los años 90, la Organización Mundial de la Salud (OMS) estableció estándares recomendados que relacionan la superficie de áreas verdes con los habitantes que la usan. De esta forma, establece un umbral mínimo de $9 \mathrm{~m}^{2} /$ habitante, óptimo de $15 \mathrm{~m}^{2} /$ habitante y máximo de $50 \mathrm{~m}^{2} /$ habitante.

Diferentes autores (Loizu, 1980; De Esteban Alonso et ÁL, 1982) han recopilado índices establecidos en distintas normativas europeas. Estas definen estándares asociados con tres categorías de espacios verdes: parques urbanos, parques suburbanos y jardines. En torno de parques urbanos se observan ciudades con estándares altos, como Viena, Milán y Estocolmo, con $25 \mathrm{~m}^{2}$ /habitante; otras con intermedios, como París, Berlín, Londres y Ámsterdam, que tienen entre 5 y $15 \mathrm{~m}^{2} /$ habitante, y otras con más bajos, como Hamburgo, con 2-4 m²/ habitante. Según lo que presentan De Esteban Alonso ET ÁL, (1982), los límites de estos estándares pueden tener variaciones significativas para las distintas categorías de espacio verde. Si se consideran las propuestas para parques urbanos y jardines, el valor mínimo es de $3 \mathrm{~m}^{2}$ suelo/habitante, mientras que el máximo es de $13 \mathrm{~m}^{2}$ suelo/habitante.

Latinoamérica —y de modo particular Argentina, PANASITI (1994)— ha desarrollado un índice que determina los requerimientos de espacios verdes asociados a áreas construidas, y establece una relación óptima de 1: 4. Este criterio persigue configurar relaciones porcentuales entre el ambiente natural y el ambiente artificial. La aplicación de este indicador a un conjunto de ciudades europeas y americanas indica la necesidad de duplicar los espacios verdes en Madrid, Bilbao, Ámsterdam y Chicago y cuadriplicarlos en ciudades americanas como México y Mendoza (Del Saz Salazar et ÁL., 2007; NowaK eT aL., 1993). Sin embargo, estos índices discutidos determinan cantidad de espacios verdes por habitante, pero no tienen en cuenta sus características respecto de su distribución, diseño, materialidad y las proporciones de árboles, arbustos y césped involucradas.

\section{Modelo urbano de ciudades emplazadas en zonas áridas}

La concepción urbanística de las ciudades responde a dos modelos de organización físicoespacial: un primer modelo "compacto", en el cual domina el uso intensivo del espacio y la diversidad de funciones y se propicia la concentración poblacional en áreas de altas densidades y la mezcla de usos. Su desarrollo urbano es continuo, basado en la compacidad de 
formas, combinando trazas orgánicas y racionales. Por otro lado, el modelo de desarrollo “disperso" y extendido, nacido a partir de los procesos de industrialización y del uso del automóvil, en el cual el centro urbano cumple funciones administrativas, financieras y comerciales, se relaciona mediante carreteras con centros secundarios industriales, comerciales y extensas áreas residenciales periféricas.

Particularmente en contextos áridos, donde las condiciones climáticas son restrictivas, el modelo "compacto" es el que predomina, como es el caso de la ciudad de Argelia. Presenta calles estrechas y edificios entrelazados por patios de reducidas dimensiones. El modelo en sí mismo construye sombras que disminuyen la exposición solar en la estación cálida y, en consecuencia, la acumulación de calor sobre las superficies duras. La volumetría arquitectónica se organiza en torno de una plaza principal, del tipo "seca”, donde la presencia de espacios verdes es de carácter limitada o nula (ver figura 1).

A diferencia de otras ciudades emplazadas en contextos áridos, la ciudad de Mendoza, en Argentina, muestra un modelo de desarrollo del tipo "disperso", definido por calles anchas y edificios contenidos en una trama en damero. La ciudad presenta numerosos espacios verdes, y sus calles están flanqueadas en sus límites por líneas de árboles que producen sombras sobre las superficies urbanas. Esta estrategia minimiza la exposición solar del conjunto y constituye el soporte del modelo (ver figura 1).

\section{Mendoza, “Ciudad Oasis"}

La ciudad de Mendoza está emplazada en el centro oeste de la República Argentina, a 32 40' Latitud Sur, 68 $51^{\prime}$ Longitud Oeste y 750 msnm (metros sobre el nivel del mar). Su estructura urbana resulta de la superposición de tres tramas: una primera de manzanas en damero de $100 \mathrm{~m}$ por $100 \mathrm{~m}$, que contiene la volumetría arquitectónica articulada mediante patios; la segunda corresponde a calles y veredas anchas de 16, 20 y 30 metros, fuertemente forestadas y la tercera, una trama verde compuesta por parques, plazas y arbolado de alineación en calles - 700.000 ejemplares en el área metropolitana— (MARTíneZ ET ÁL., 2009). Este modelo de desarrollo es consecuencia del proceso de reconstrucción de la ciudad nueva en 1863, después de un fuerte sismo que desbastó la ciudad colonial (1861) (PonTE, 2008). La reconstrucción de la ciudad priorizó los aspectos de orden administrativo, funcional y paisajístico (BormidA, 1984; MoRETTI, 2010). 
Espacios verdes en ciudades de zona árida.

Diagnóstico de la situación actual de plazas de la ciudad de Mendoza, Argentina

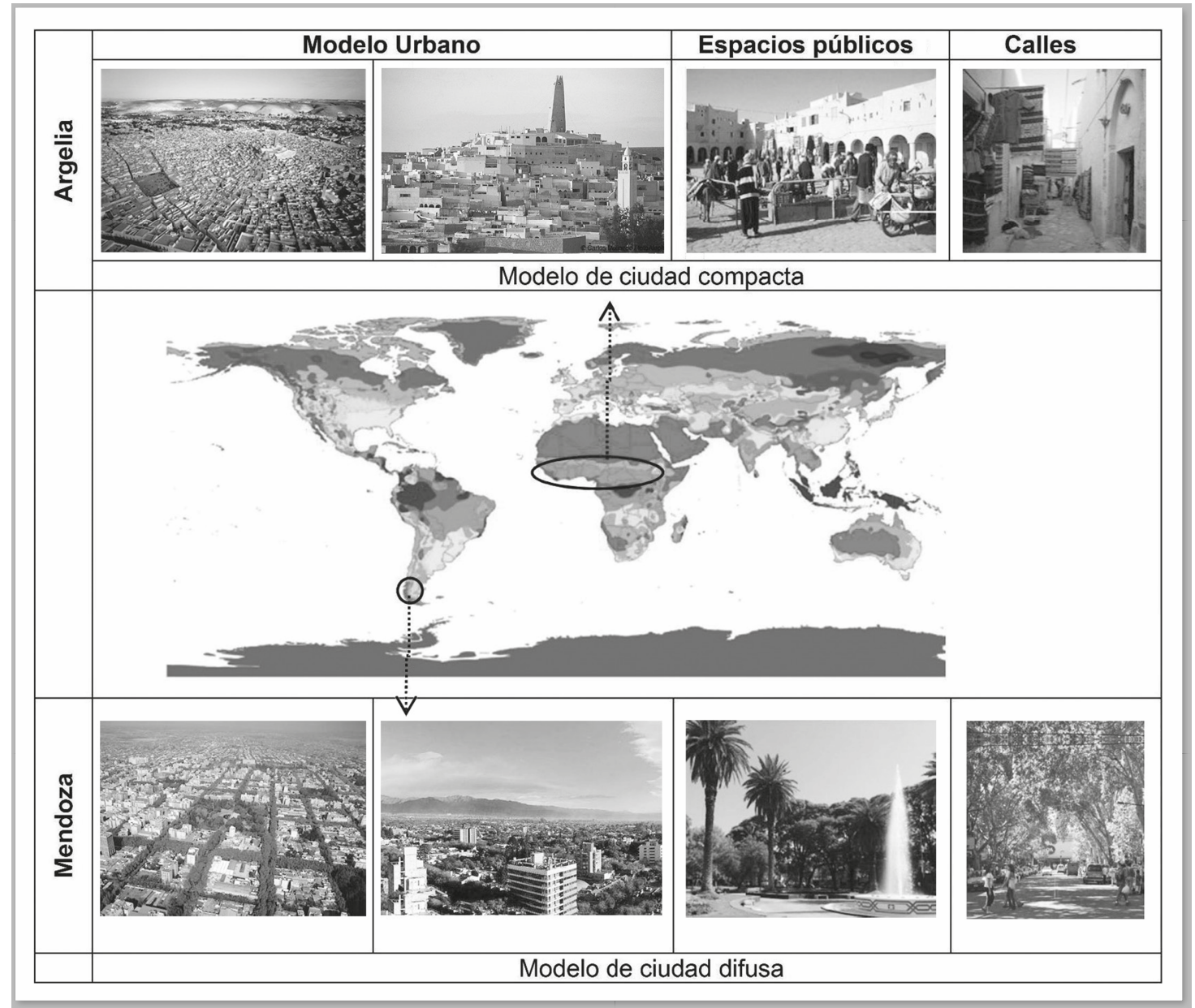

Figura 1. Modelos urbanos de ciudades áridas Fuente: elaboración propia 
Se organizó en damero de 64 manzanas, con una plaza central de cuatro hectáreas y en las diagonales, de forma equidistante al foco principal, cuatro plazas de una hectárea cada una (MoRETTI, 2010). La ciudad nació y creció con un fuerte contenido de espacios verdes, presentando una relación de 64 manzanas construidas y ocho destinadas a plazas, que dio lugar a un modelo denominado internacionalmente como "Ciudad Oasis" (BormidA, 1984) (ver figura 2).

En la actualidad los espacios verdes coexisten armónicamente en dos categorías: en la trama urbana global a lo largo de calles, plazas y parques, y el nivel de detalle localizado, es decir, situaciones particulares de cada manzana urbana, en donde el espacio verde es el resultante de la articulación de los volúmenes construidos. En relación con ellos, la arboleda urbana es largamente la más significativa (48.000 ejemplares) e interactúa con el ámbito construido definiendo distintas estructuras urbano-espaciales.

El área central de la ciudad muestra una estructura verde consolidada, continua, de tipo abovedada. La densidad de edificación es alta y media, con tipologías edilicias de varios niveles sobre la línea municipal. El área residencial peri-central y periférica posee una estructura verde consolidada, abierta y discontinua, con densidad de edificación media y baja. Viviendas unifamiliares y ocasionales unidades de desarrollo vertical. Por último, los conjuntos de viviendas sociales y asentamientos no planificados muestran una forestación urbana escasa o inexistente. Viviendas económicas de construcción por iniciativa estatal, gestión cooperativa o autoconstruidas.

\section{Problemática de la ciudad de Mendoza}

El desarrollo urbano del siglo XX debilitó la trama verde característica de la ciudad y, en consecuencia, el modelo, debido a un conjunto de razones de orden edilicia y urbana. Respecto de las razones edilicias, se destaca el incremento de los factores de ocupación del suelo que propone mayores densidades edilicias, resultantes del aumento en los factores de ocupación del suelo (FOS-FOT). Por otro lado, la falta de normativas que regulan el espacio abierto hacia el interior de los lotes individuales restringe el "pulmón de manzana", lo que permite el avance de entes privados sobre los límites de los espacios abiertos.

En lo que respecta a las razones de orden urbano, las plazas de la ciudad de Mendoza representaban el $12.5 \%$ de la trama urbana fundacional (de 64 manzanas fundacionales, 8 
estaban destinadas a plazas). Esta proporción de manzanas construidas-vacías destinadas a plazas no se respetó, y fue disminuyendo con el tiempo; en la actualidad solo el $2 \%$ de la trama urbana está destinado a plazas (Stocco ET ÁL., 2013). Por otra parte, la modernización de plazas —en el período comprendido entre 1980 y 1990 - responde a nuevas pautas de diseño y materialización del espacio abierto, donde se tiende a incorporar una gran proporción de áreas "muertas" con materiales diversos, como cemento alisado, adoquines, revestimientos calcáreos, etc. Esto altera la condición "natural" de los espacios abiertos, al disminuir las áreas vegetadas.

En consecuencia, el debilitamiento de los espacios verdes no solo empobreció las condiciones de habitabilidad de la ciudad, sino que trajo aparejada la búsqueda de nuevas formas de vida, más asociadas con la naturaleza. Se ha desarrollado una periferia residencial de casas individuales ajardinadas, de baja altura, con tendencia a la horizontalidad. El resultado de este crecimiento urbano llevó a que la ciudad adoptara el modelo de ciudad "difusa” y difícilmente sostenible. Esta

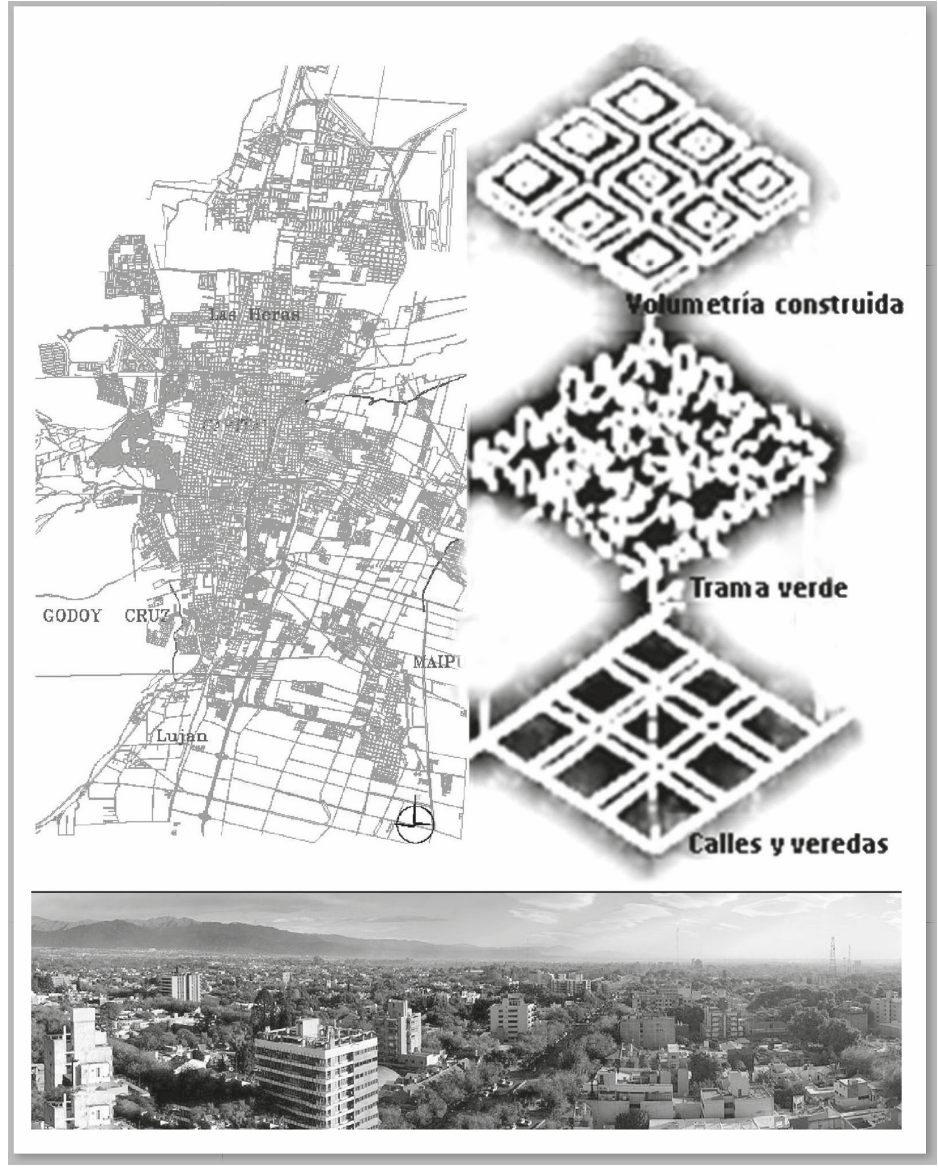

Figura 2. Modelo urbano adoptado por la ciudad de Mendoza Fuente: elaboración propia. Basado en gráficos elaborados por BORMIDA (1984) 
modalidad de desarrollo es aún más crítica en el caso de la ciudad de Mendoza, localizada en una zona árida donde los suelos productivos corresponden a los oasis sobre los cuales crece la ciudad limitando el recurso.

Revertir los fenómenos descritos implica retomar la estrategia del enverdecimiento y proponer un diseño eficiente de las áreas verdes que maximice sus beneficios ambientales teniendo en cuenta las condicionantes y limitaciones que presenta una ciudad inserta en zonas áridas: déficit hídrico y altos niveles de heliofanía. De esta forma, se encauzará el desarrollo urbano de modo de garantizar no solo la calidad ambiental y la habitabilidad del medio, sino también apuntar al bienestar social de sus habitantes.

A partir de la problemática descrita, este trabajo analiza la configuración morfológica y la materialidad de las plazas atendiendo al carácter relevante de aquella en la estructura urbana de la ciudad. Se quiere elaborar un diagnóstico actual con el objetivo final de definir pautas de diseño tendientes a maximizar beneficios ambientales y sociales que sean de utilidad a diseñadores y proyectistas.

\section{Importancia de las plazas en la trama urbana}

Localmente se entiende a la plaza como un espacio urbano público, al aire libre, en el cual se desarrollan diversas actividades. Las plazas principales forman parte del centro de la ciudad y determinan el plano físico de la trama urbana en términos de importancia histórica, ya que es el lugar donde se fundó la ciudad y la zona donde se desarrollan las actividades más importantes: económicas, políticas y administrativas. Estos espacios presentan diversas características que se fueron modificando a lo largo del tiempo. Es necesario diferenciar tres estilos que se destacan a nivel mundial, respecto de su concepción morfológica, espacial y material. En primer lugar, el modelo de plaza seca, denominado estilo europeo, en el cual el rasgo dominante es el espacio abierto y la condición sellada, con especies arbóreas aisladas o estructuras artificiales de sombreo distribuidas generalmente en forma regular. Por otro lado, las plazas de ciudades norteamericanas se caracterizan como una "explanada” delimitada por edificios. Por último, el modelo de plazas latinoamericano correspondiente a ciudades fundadas por los españoles responde al concepto de plaza jardín, donde la vegetación toma un papel importante en el diseño combinando 
Espacios verdes en ciudades de zona árida. Diagnóstico de la situación actual de plazas de la ciudad de Mendoza, Argentina

distintas categorías de vegetación como grupos de árboles de diferentes características, prados y superficies de material alternadas.

El modelo de plaza adoptado en la ciudad de Mendoza responde al concepto de plaza jardín. Son relevantes a nivel paisajístico, ya que generan espacios con recorridos y áreas de estancia que discontinúan los perfiles construidos. Generalmente incorporan la vegetación como elemento de diseño, y en segundo plano aparecen objetos decorativos como fuentes con agua, monumentos, etc., que permiten la transición entre arquitectura y naturaleza. Socialmente las plazas son espacios de encuentro, donde la población se reúne para conmemorar acontecimientos históricos y celebraciones; también se desarrollan diversas actividades recreativas, deportivas, educativas, entre otras.

Estos espacios vegetados son significativos por su frecuencia y distribución en la trama urbana. En la actualidad poseen una atención especial, por producir efectos positivos en el microclima local que son dependientes de las características de diseño de la plaza, en relación con su distribución formal, características y proporciones de la vegetación y materialidad (STоссо ET ÁL., 2015).

\section{Caracterización de distribución y dimensiones}

Las plazas resultan relevantes respecto del total de espacios verdes presentes en la trama urbana de la ciudad: representan $212549 \mathrm{~m}^{2}$ y determinan el $4.43 \%$ haciendo diferencia entre parques, plazas, plazoletas y paseos. La ciudad contiene catorce plazas que representan el 30 $\%$ del total de los espacios verdes. Alcanzan dimensiones promedio de $10.000 \mathrm{~m}^{2}$; este rango está determinado por su inserción en la trama en damero de $100 \mathrm{~m}$ por $100 \mathrm{~m}$. Respecto de sus dimensiones máximas, corresponde a la plaza principal, plaza Independencia, con 47357.9 $\mathrm{m}^{2}$, y las dimensiones mínimas se dan en la plaza Manuel Belgrano, de 7358,5 $\mathrm{m}^{2}$ que resultan del crecimiento de la ciudad asociada a otras morfologías de manzana urbana.

Su distribución en la trama responde a dos criterios: respecto del área central se presenta de forma homogénea asociada con un esquema de plaza central y plazas equidistantes; en relación con las plazas ubicadas en la periferia del microcentro, su distribución es aleatoria, sin responder a un patrón de distribución preestablecido en la estructura urbana (ver figura 3). 


\section{Susana Stocco, María Alicia Cantón y Erica Correa}

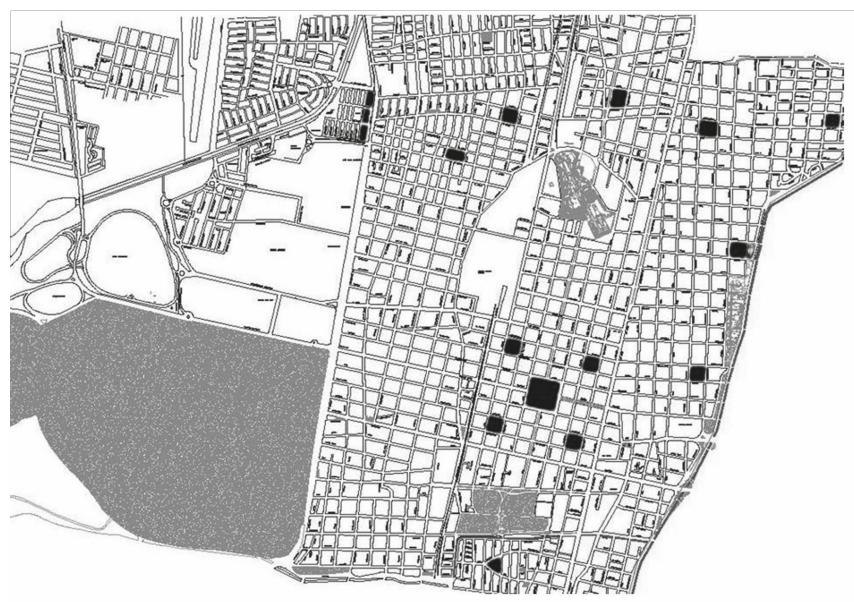

Figura 3. Distribución de las plazas de la ciudad de Mendoza Fuente: elaboración propia, sobre relevamiento satelital

\section{Clasificación de los casos de estudio}

La clasificación de los casos de estudio se realizó siguiendo parámetros físicos y sociales. En lo que respecta a los aspectos físicos de las plazas, se clasificaron según su geometría (cuadrada, rectangular, poligonal o triangular) y sus dimensiones (tamaño y proporción). Otro aspecto relevante que se tuvo en cuenta es el diseño, ya que se determinó la organización espacial interna (lineal, central, radial, simétrica y centro focal). El parámetro social evaluado se diferenció teniendo en cuenta la actividad que se realiza en la plaza (Plaza Mayor, Memorial, Corporativa, Vecinal, Mercado) y el estilo que la identifica (francés, español, etc.). A continuación se organizó y clasificó en la tabla 1 cada caso de estudio según sus características.

Como se ve en la tabla 1, podemos diferenciar la plaza Independencia con características de plaza mayor. Como se nombró previamente, forma parte del conjunto de cinco plazas que delimitan el centro de la ciudad. Está conformada por cuatro manzanas $\left(47356.9 \mathrm{~m}^{2}\right)$. La estructura espacial interna se define a partir de un centro focal configurado por una fuente de agua semicircular, a partir de la cual se organizan espacios verdes y grandes explanadas destinadas a celebraciones públicas.

Por otro lado, se puede identificar como plazas vecinales un número mayor de casos, como son las plazas Manuel Belgrano, Mathus Hoyos, Cobos, Malvinas Argentinas, entre otras, que se localizan en las áreas residenciales de la ciudad. La superficie varía entre 2500 y $10000 \mathrm{~m}^{2}$. En términos de diseño, generalmente las plazas barriales responden a la estructura espacial donde hay un centro focal y espacios verdes organizados en torno de ejes. Se diferencian de las plazas del microcentro con respecto al carácter: la concepción monumental cede el paso a un ámbito destinado a usos familiares de recreación y expansión. La plaza constituye el espacio verde de expansión de los usos habitacionales. 
Espacios verdes en ciudades de zona árida.

Diagnóstico de la situación actual de plazas de la ciudad de Mendoza, Argentina

\begin{tabular}{|c|c|c|c|c|c|c|c|c|}
\hline Tabla 1 & \multicolumn{8}{|c|}{ Fuente: elaboración propia } \\
\hline \multicolumn{9}{|c|}{ Clasificación y caracterización de cada plaza } \\
\hline \multirow{2}{*}{ Plaza } & \multirow{2}{*}{ Geometría } & \multicolumn{2}{|c|}{ Dimensiones } & \multicolumn{5}{|c|}{ Organización espacial } \\
\hline & & $\underset{\mathrm{M}^{2}}{\operatorname{Tamaño~}}$ & Proporción & $\begin{array}{l}\text { Org. } \\
\text { Interna }\end{array}$ & $\begin{array}{l}\text { Trama } \\
\text { Urbana }\end{array}$ & Tipo & Estilo & $\begin{array}{l}\text { Carac- } \\
\text { terísticas }\end{array}$ \\
\hline \multicolumn{9}{|l|}{ Chile } \\
\hline & Cuadrada & $12.081,6$ & $1 / 1$ & Radial & $\begin{array}{l}\text { Nor } \\
\text { Este }\end{array}$ & $\begin{array}{c}\text { Plaza } \\
\text { Memorial }\end{array}$ & Francés & $\begin{array}{l}\text { Plaza } \\
\text { Jardín }\end{array}$ \\
\hline \multirow[t]{2}{*}{ Cobos } & & & & & & & & \\
\hline & Poligonal & $14.406,5$ & $1 / 1$ & Radial & $\begin{array}{l}\text { Nor } \\
\text { Este }\end{array}$ & $\begin{array}{c}\text { Plaza } \\
\text { de Barrio } \\
\text { Memorial }\end{array}$ & Francés & $\begin{array}{l}\text { Plaza } \\
\text { Jardín }\end{array}$ \\
\hline \multirow{2}{*}{ Dr. E. Matons } & \multirow[b]{2}{*}{ Rectangular } & \multirow[b]{2}{*}{9620,7} & \multirow[b]{2}{*}{$1 / 1$} & \multirow[b]{2}{*}{ Central } & \multirow[b]{2}{*}{$\begin{array}{l}\text { Nor } \\
\text { Este }\end{array}$} & \multirow[b]{2}{*}{$\begin{array}{c}\text { Plaza } \\
\text { Memorial }\end{array}$} & \multirow[b]{2}{*}{ Francés } & \multirow[b]{2}{*}{$\begin{array}{l}\text { Plaza } \\
\text { Jardín }\end{array}$} \\
\hline & & & & & & & & \\
\hline \multirow[t]{2}{*}{ España } & \multirow[b]{2}{*}{ Cuadrada } & \multirow[b]{2}{*}{$12.034,5$} & \multirow[b]{2}{*}{$1 / 1$} & \multirow[b]{2}{*}{ Central } & \multirow[b]{2}{*}{$\begin{array}{l}\text { Nor } \\
\text { Este }\end{array}$} & \multirow[b]{2}{*}{$\begin{array}{c}\text { Plaza } \\
\text { Memorial }\end{array}$} & \multirow[b]{2}{*}{ Español } & \multirow[b]{2}{*}{$\begin{array}{l}\text { Plaza } \\
\text { Jardín }\end{array}$} \\
\hline & & & & & & & & \\
\hline \multirow{2}{*}{ Independencia } & \multirow[b]{2}{*}{ Cuadrada } & \multirow[b]{2}{*}{$12.081,6$} & \multirow[b]{2}{*}{$1 / 1$} & \multirow[b]{2}{*}{ Central } & \multirow[b]{2}{*}{$\begin{array}{l}\text { Nor } \\
\text { Este }\end{array}$} & \multirow[b]{2}{*}{$\begin{array}{c}\text { Plaza } \\
\text { Mayor } \\
\text { Parque } \\
\text { Urbano }\end{array}$} & \multirow[b]{2}{*}{ Francés } & \multirow[b]{2}{*}{$\begin{array}{l}\text { Plaza } \\
\text { Jardín }\end{array}$} \\
\hline & & & & & & & & \\
\hline \multirow[t]{2}{*}{ Italia } & & & & & & & & \\
\hline & Cuadrada & $14.406,5$ & $1 / 1$ & Radial & $\begin{array}{l}\text { Nor } \\
\text { Este }\end{array}$ & $\begin{array}{c}\text { Plaza } \\
\text { Memorial }\end{array}$ & Francés & $\begin{array}{l}\text { Plaza } \\
\text { Jardín }\end{array}$ \\
\hline Irigoyen & & & & & & & & \\
\hline 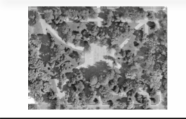 & Rectangular & 9620,7 & $1 / 1$ & Radial & $\begin{array}{l}\text { Nor } \\
\text { Este }\end{array}$ & $\begin{array}{c}\text { Plaza } \\
\text { Memorial }\end{array}$ & Francés & $\begin{array}{l}\text { Plaza } \\
\text { Jardín }\end{array}$ \\
\hline
\end{tabular}




\section{Susana Stocco, María Alicia Cantón y Erica Correa}

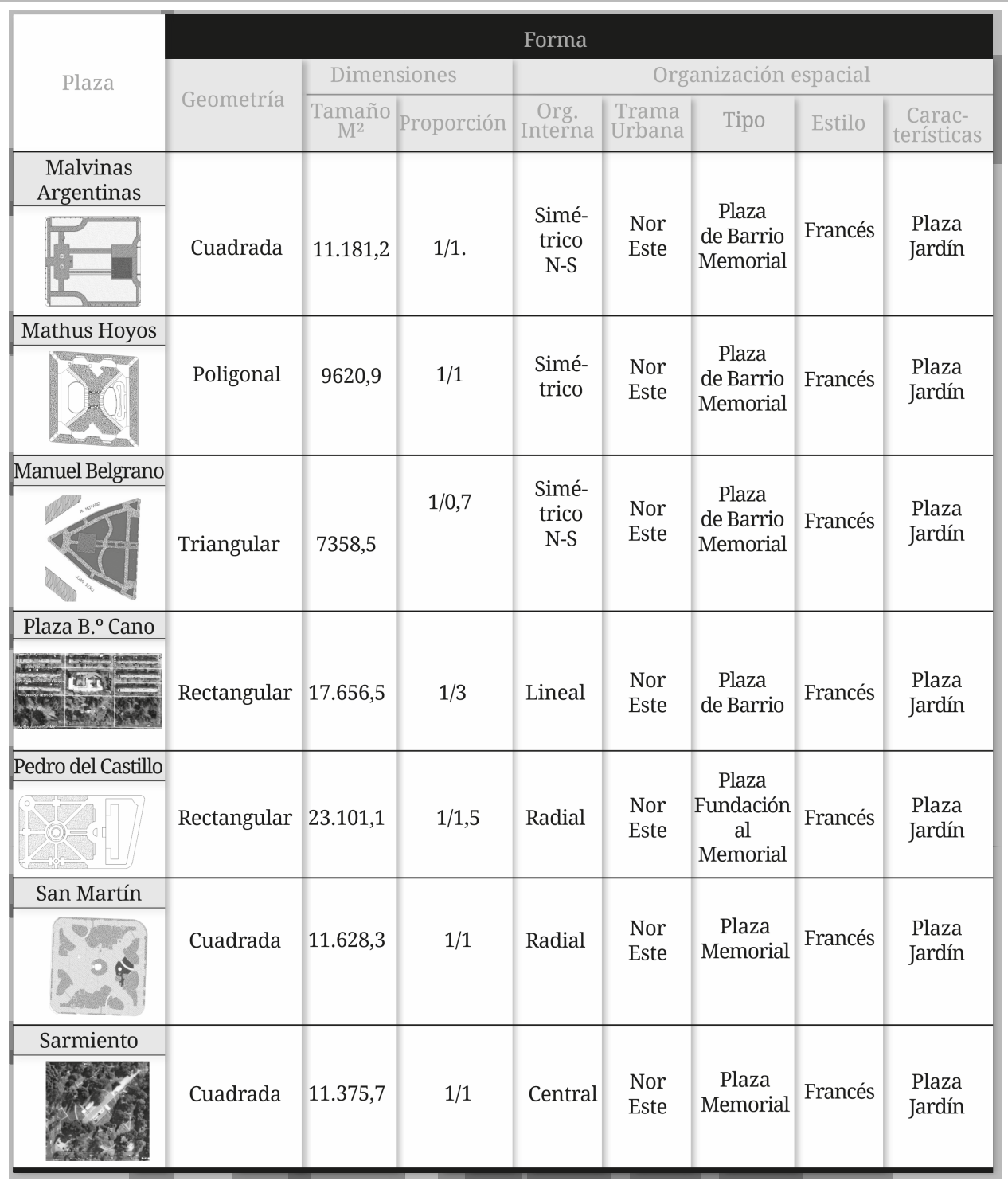


Espacios verdes en ciudades de zona árida.

Diagnóstico de la situación actual de plazas de la ciudad de Mendoza, Argentina

En todos los casos revisten un carácter memorial, ya que contienen elementos referentes de hechos históricos o rinden honores a figuras de elevada trayectoria para la sociedad. Desde el punto de vista del diseño, la gran mayoría de las plazas son de forma cuadrada, ya que responden a la traza en damero de la ciudad. Representan el $50 \%$, mientras que un $29 \%$ corresponde a plazas rectangulares derivadas de la modificación de la trama urbana, un 14 $\%$ a poligonales y un $7 \%$ a triangulares resultantes de la vinculación física de tramas con diferente orientación (ver figura 4).

La concepción del espacio es de carácter simétrico, organizado en torno de ejes que toman como punto de partida un núcleo que marca la centralidad del espacio abierto. Dicho centro está formado generalmente por una explanada que contiene espejos de agua y monumentos. Los ejes, de tipo radial, definen áreas verdes conformadas por extensiones de prados y macizos definidos por el agrupamiento de especies vegetales de diferente magnitud. En el $78.5 \%$ de las plazas domina la condición verde debido al fuerte contenido de espacios vegetados. Se trata de "plazas jardín". En un 14.3 \% de las plazas dominan las superficies selladas y en un 7,14 \% se observa un equilibrio entre áreas verdes y selladas. Esta condición es el resultado del proceso de modernización de muchas de ellas, que ha debilitado la presencia de áreas verdes (ver figura 5 y tabla 2).

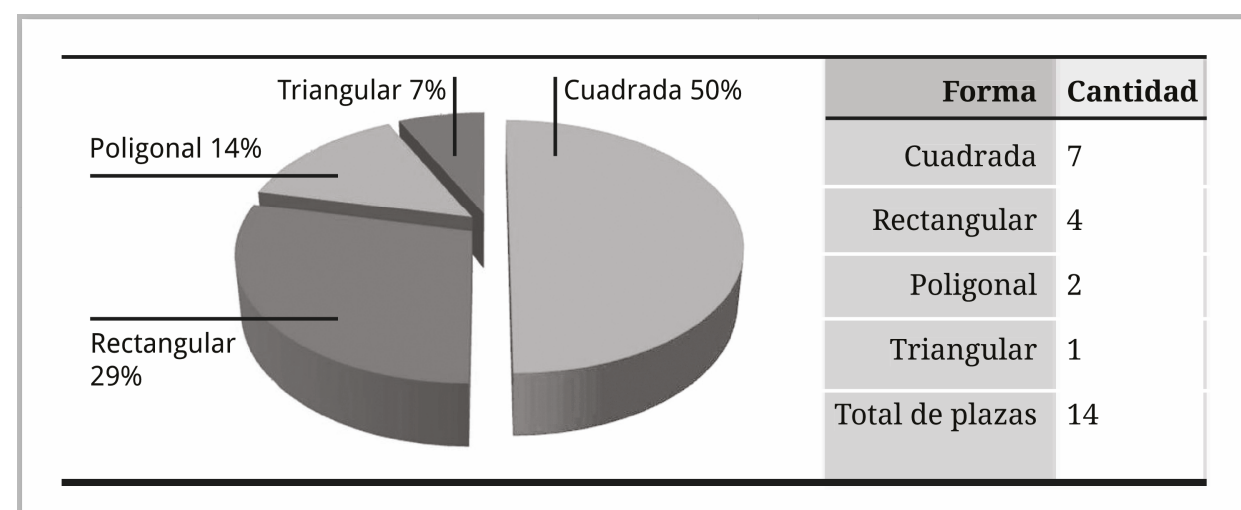

Figura 4. Distribución porcentual de las plazas de acuerdo con su forma Fuente: elaboración propia 


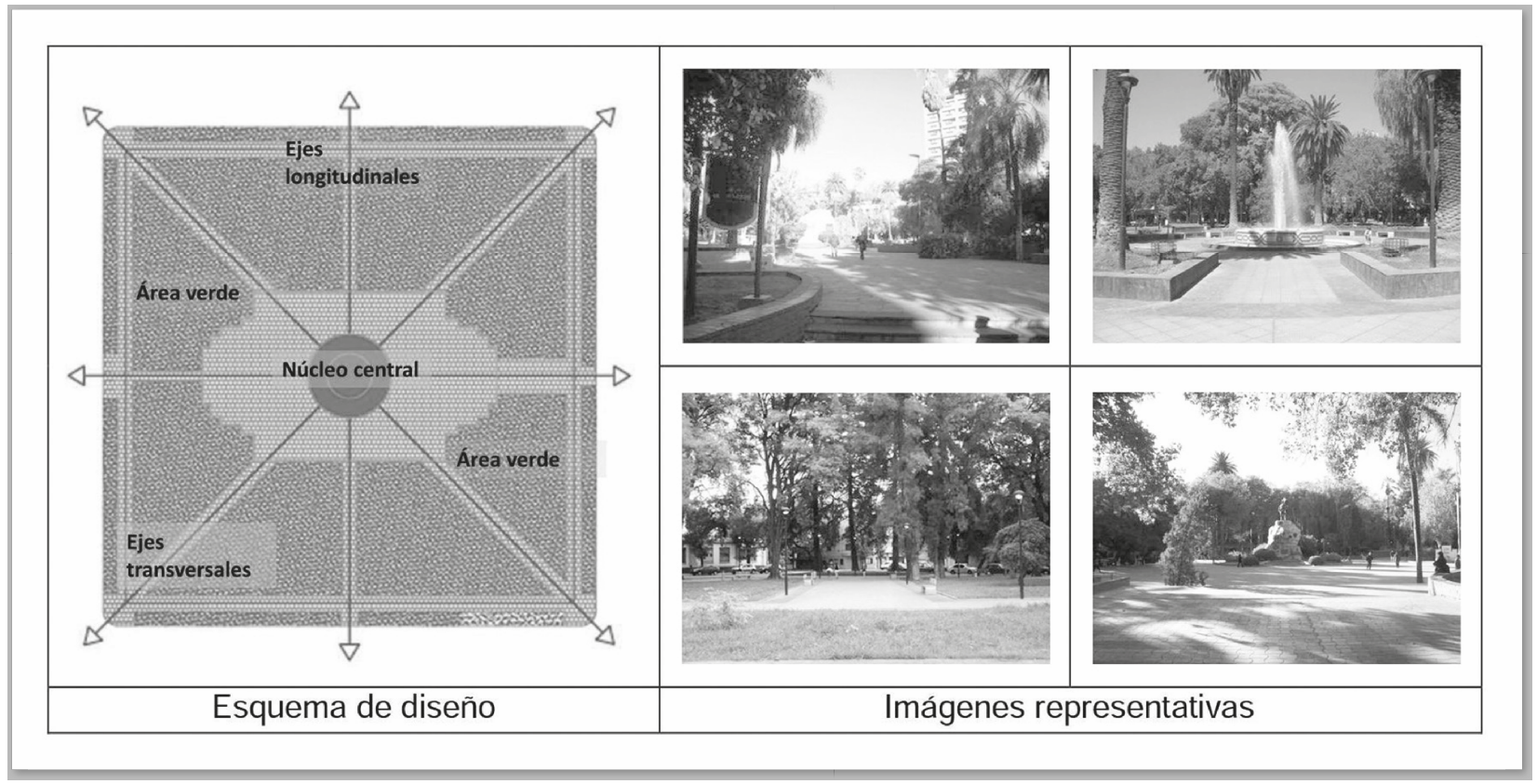

Figura 5. Esquema de diseño típico de las plazas en Mendoza Fuente: elaboración propia
Figura 6. Distribución porcentual de las plazas de Mendoza de acuerdo con su materialidad Fuente: elaboración propia

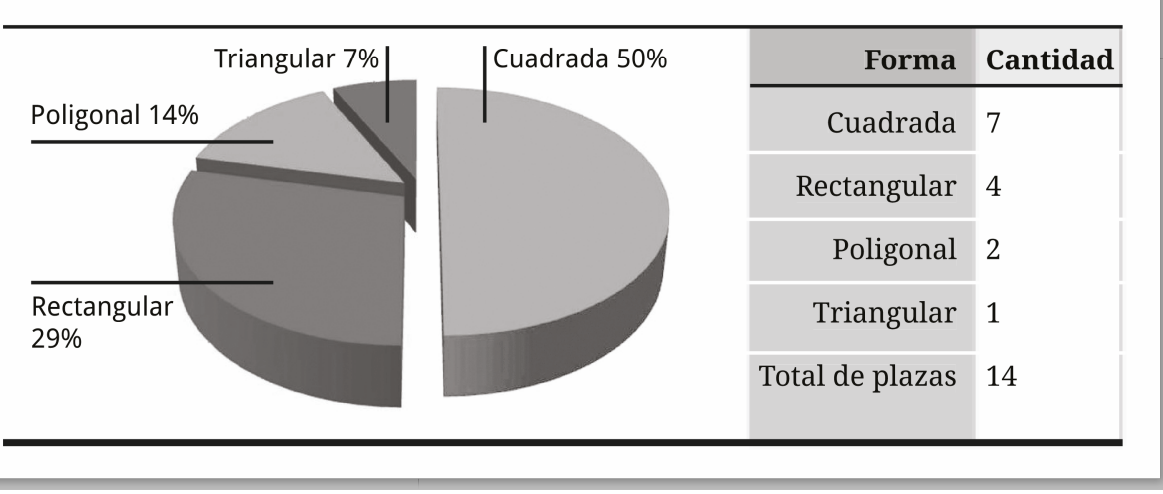


Espacios verdes en ciudades de zona árida.

Diagnóstico de la situación actual de plazas de la ciudad de Mendoza, Argentina

\section{Tabla 2}

\section{Clasificación de cada plaza respecto de su materialidad}

\begin{tabular}{|c|c|c|c|c|c|c|c|}
\hline \multirow[t]{2}{*}{ Plaza } & \multicolumn{3}{|c|}{ Superficies internas } & \multicolumn{4}{|c|}{ Materialidad } \\
\hline & Sup. total & Largo & Ancho & \%Verde & \%Pisos & \%Otros & Otros \\
\hline Chile & 12081.6 & 111 & 109 & 51 & 49 & 0.44 & Agua \\
\hline Cobos & 14406.5 & 126 & 109.32 & 59.77 & 40.22 & - & - \\
\hline Dr. E. Matons & 10640.9 & 77.57 & 139.03 & 65 & 35 & - & - \\
\hline España & 12034.5 & 110 & 109 & 55.34 & 44.29 & 0.37 & Agua \\
\hline Independencia & 47356.9 & 217.65 & 217.65 & 49 & 49 & 2.15 & Agua \\
\hline Italia & 12234.5 & 111.3 & 110 & 48.99 & 50.43 & 0.56 & Agua \\
\hline Irigoyen & 19913.4 & 142.79 & 114.73 & 65 & 35 & - & - \\
\hline $\begin{array}{l}\text { Malvinas } \\
\text { Argentinas }\end{array}$ & 11181.2 & 110 & 101.8 & 59 & 41 & - & - \\
\hline Mathus Hoyos & 9620.9 & 98.83 & 96.22 & 52 & 40 & 8 & Tierra \\
\hline Manuel Belgrano & 7358.5 & 165.71 & 192.27 & 54 & 12 & 35 & Ladrillo molido \\
\hline Plaza B ${ }^{\circ}$ Cano & 37324.5 & 389.18 & 83.55 & 60 & 5.8 & 34.22 & Tierra \\
\hline \multirow[t]{2}{*}{ Pedro del Castillo } & \multirow[t]{2}{*}{23104.4} & \multirow[t]{2}{*}{180} & \multirow[t]{2}{*}{111.97} & \multirow[t]{2}{*}{45} & \multirow[t]{2}{*}{42} & 8 & Edificación \\
\hline & & & & & & 5 & Agua \\
\hline San Martín & 11699.8 & 109.19 & 108.05 & 43 & 57 & 0.22 & Agua \\
\hline Sarmiento & 12224.6 & 111.5 & 110.16 & 59 & 41 & - & - \\
\hline
\end{tabular}

Fuente: elaboración propia

\section{Contexto de inserción}

La ciudad de Mendoza presenta un esquema de distribución edilicio del tipo piramidal. Es decir, máxima concentración de edificios — de más de seis pisos de altura - en el centro de la ciudad, que se diluye hacia la periferia. En ella se desarrollan áreas residenciales: viviendas y conjuntos de viviendas de uno a tres niveles de altura. Entre ambas densidades, se identifica un área de transición, de densidad intermedia, conformada por edificios de tres a seis niveles. 
El esquema descrito resulta del proceso de desarrollo de la ciudad: una primera etapa espontánea que se corresponde con la fundación y una segunda etapa regulada por el código de edificación de la ciudad de Mendoza a través de la Ordenanza 3866/2014 (Municipalidad de Mendoza, 2014), en la que se establecen zonas y se clasifican los usos del suelo. En ella se identifican las siguientes categorías:

- Zona central y comercial: son zonas de media-alta densidad, destinadas a la localización predominante de usos administrativos, institucionales, financieros, comerciales y de servicios a distintos niveles cualitativos y cuantitativos, que definen rasgos diferenciales entre las distintas categorías.

- Zona residencial: son zonas destinadas a la preservación de sus características residenciales, cuya morfología urbana se caracteriza por una trama de calles predominantemente ortogonales con manzanas cuadradas o rectangulares, con condiciones de barrio jardín.

En este contexto edilicio se insertan las catorce plazas, de las cuales el $21.4 \%$ se encuentran en alta densidad, ubicadas en el centro de la ciudad; el $14.3 \%$ corresponde a media densidad edilicia, con el modelo de plaza principal, y cuatro plazas equidistantes. Por último, el $64.3 \%$ se encuentra en baja densidad edilicia. Esta situación se da porque del total de la trama urbana de la ciudad de Mendoza, solo el $17 \%$ corresponde a alta-media densidad edilicia y el $83 \%$, a baja densidad edilicia. Respecto de la zonificación de actividades, el 42.9 $\%$ de las plazas se encuentra en zona residencial, el $21.4 \%$ en zona comercial y el $35.7 \%$ en zona residencial mixta (ver tabla 3).

Se puede distinguir en cuanto al contexto de inserción que las plazas se insertan en alta, media y baja densidad edilicia, sin mostrar relación de dependencia con la zonificación de la ciudad de acuerdo con los usos del suelo.

\section{Consideraciones finales}

La ciudad de Mendoza, a diferencia de otras ciudades emplazadas en contextos áridos, presenta un modelo de desarrollo urbano que difiere de la estructura urbano-espacial dominante en las zonas áridas. Su concepción urbanística se basa en la presencia de vegetación como estrategia de sombreo, que minimiza la exposición solar del conjunto y mejora la habitabilidad de los espacios al aire libre. 
Espacios verdes en ciudades de zona árida.

Diagnóstico de la situación actual de plazas de la ciudad de Mendoza, Argentina

\begin{tabular}{|c|c|c|c|c|c|c|}
\hline Tabla 3 & \multicolumn{6}{|c|}{ Fuente: elaboración propia } \\
\hline \multicolumn{7}{|c|}{ Clasificación según el contexto de inserción } \\
\hline Plaza & \multicolumn{2}{|c|}{ Ubicación } & Densidad & Configu- & Orientación & Zona \\
\hline \multirow{3}{*}{ 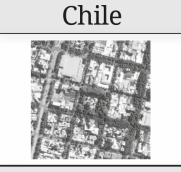 } & Latitud & $32^{\circ} 53^{\prime} 10.74 "$ & \multirow{3}{*}{ Media } & \multirow{3}{*}{ Ortogonal } & \multirow{3}{*}{$92^{\circ}$} & \multirow{3}{*}{$\begin{array}{l}\text { Comercial } \\
\text { Mixta }\end{array}$} \\
\hline & Longitud & $68^{\circ} 50^{\prime} 47.31$ & & & & \\
\hline & Altitud & 770 & & & & \\
\hline \multirow{3}{*}{ 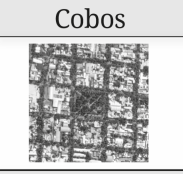 } & Latitud & $32^{\circ} 52^{\prime} 16.87^{\prime \prime}$ & \multirow{3}{*}{ Baja } & \multirow{3}{*}{ Ortogonal } & \multirow{3}{*}{$86^{\circ}$} & \multirow{3}{*}{ Residencial } \\
\hline & Longitud & $68^{\circ} 49^{\prime} 52.27^{\prime \prime}$ & & & & \\
\hline & Altitud & 739 & & & & \\
\hline \multirow{3}{*}{ 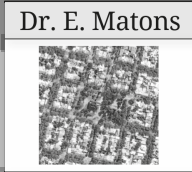 } & Latitud & $32^{\circ} 52^{\prime} 23.37^{\prime \prime}$ & \multirow{3}{*}{ Baja } & \multirow{3}{*}{ Ortogonal } & \multirow{3}{*}{$79^{\circ}$} & \multirow{3}{*}{ Residencial } \\
\hline & Longitud & $68^{\circ} 51^{\prime} 06.11^{\prime \prime}$ & & & & \\
\hline & Altitud & 768 & & & & \\
\hline \multirow{3}{*}{ 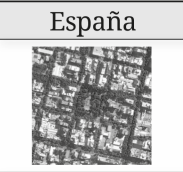 } & Latitud & $32^{\circ} 53^{\prime} 33.33^{\prime \prime}$ & \multirow{3}{*}{ Alta } & \multirow{3}{*}{ Ortogonal } & \multirow{3}{*}{$98^{\circ}$} & \multirow{3}{*}{ Comercial } \\
\hline & Longitud & $68^{\circ} 50^{\prime} 30.23^{\prime \prime}$ & & & & \\
\hline & Altitud & 771 & & & & \\
\hline \multirow{3}{*}{ 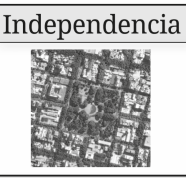 } & Latitud & $32^{\circ} 53^{\prime} 28.70^{\prime \prime}$ & \multirow{3}{*}{ Alta } & \multirow{3}{*}{ Ortogonal } & \multirow{3}{*}{$78^{\circ}$} & \multirow{3}{*}{$\begin{array}{l}\text { Comercial } \\
\text { Mixta }\end{array}$} \\
\hline & Longitud & $68^{\circ} 50^{\prime} 40.33$ & & & & \\
\hline & Altitud & 769 & & & & \\
\hline \multirow{4}{*}{ 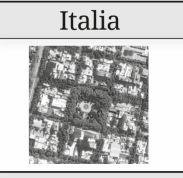 } & Latitud & $32^{\circ} 53^{\prime} 3153^{\prime \prime}$ & \multirow{4}{*}{ Alta } & \multirow{4}{*}{ Ortogonal } & \multirow{4}{*}{$78^{\circ}$} & \multirow{4}{*}{ Residencial } \\
\hline & I ancituld & & & & & \\
\hline & Longitua & $68^{\circ} 50$ 51.49” & & & & \\
\hline & Altitud & 778 & & & & \\
\hline Irigoyen & Latitud & $32^{\circ} 52^{\prime} 9.38^{\prime \prime}$ & & & & \\
\hline 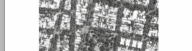 & Longitud & $68^{\circ} 50^{\prime} 18.45^{\prime \prime}$ & Ваја & Ortogonal & $74^{\circ}$ & Residencial \\
\hline Broments & Altitud & 748 & & & & \\
\hline
\end{tabular}




\begin{tabular}{|c|c|c|c|c|c|c|}
\hline & & & & Tram & Urbana & \\
\hline Plaza & & ción & Densidad & Configu- & $\begin{array}{l}\text { Orientación } \\
\text { de la Trama }\end{array}$ & Zona \\
\hline Malvinas Argentinas & Latitud & $32^{\circ} 53^{\prime} 10.74 "$ & & & & \\
\hline Es: & Longitud & $68^{\circ} 50^{\prime} 47.31$ & Baja & Ortogonal & $78^{\circ}$ & Residencial \\
\hline ser evi: & Altitud & 770 & & & & \\
\hline Mathus Hoyos & Latitud & $32^{\circ} 52^{\prime} 16.87^{\prime \prime}$ & & & & \\
\hline Wes & Longitud & $68^{\circ} 49^{\prime} 52.27^{\prime \prime}$ & Baja & Ortogonal & $84^{\circ}$ & Comercial \\
\hline syesis & Altitud & 739 & & & & \\
\hline Manuel Belgrano & Latitud & $32^{\circ} 52^{\prime} 23.37^{\prime \prime}$ & & & & \\
\hline & Longitud & $68^{\circ} 51^{\prime} 06.11^{\prime \prime}$ & Baja & Irregular & $87^{\circ}$ & Residencial \\
\hline Mta & Altitud & 768 & & & & \\
\hline Plaza B. ${ }^{\circ}$ Cano & Latitud & $32^{\circ} 53^{\prime} 33.33^{\prime \prime}$ & & & & \\
\hline & Longitud & $68^{\circ} 50^{\prime} 30.23^{\prime \prime}$ & Ваја & Ortogonal & $82^{\circ}$ & Residencial \\
\hline MII 50 & Altitud & 771 & & & & \\
\hline Pedro del Castillo & Latitud & $32^{\circ} 53^{\prime} 28.70^{\prime \prime}$ & & & & \\
\hline & Longitud & $68^{\circ} 50^{\prime} 40.33$ & Baja & Ortogonal & 90 & $\begin{array}{l}\text { Comerclal } \\
\text { Mixta }\end{array}$ \\
\hline mysula & Altitud & 769 & & & & \\
\hline San Martín & Latitud & $20^{\circ} 52^{\prime} 3152 "$ & & & & \\
\hline Mats & Longitud & $68^{\circ} 50^{\prime} 5149^{\prime \prime}$ & Alta & Ortogonal & $84^{\circ}$ & Comercial \\
\hline 6ot & Altitud & 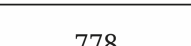 & & & & \\
\hline Sarmiento & Latitud & $32^{\circ} 52^{\prime} 9.38^{\prime \prime}$ & & & & \\
\hline Tinety & Longitud & $68^{\circ} 50^{\prime} 18.45^{\prime \prime}$ & Baja & Ortogonal & $89^{\circ}$ & Comprcisl \\
\hline Ginces? & Altitud & 748 & & & & \\
\hline
\end{tabular}


Esta trama verde característica de la ciudad de Mendoza se fue debilitado en relación con los procesos de desarrollo urbano del siglo XX; particularmente las plazas fueron perdiendo espacio en la trama urbana, ya que se priorizaron las manzanas construidas frente a las destinadas a espacios verdes. Por otro lado, se produjo también la pérdida de superficies verdes y la incorporación de materiales sellados con los cuales se remodelaban estos espacios, que los transformó en espacios agradables paisajísticamente, pero que empobrecen las condiciones de habitabilidad de la ciudad.

Este trabajo reflexiona particularmente sobre el diseño actual de "las plazas" en la ciudad de Mendoza, evaluando las características de diseño: formal, material y el contexto en el cual se insertan. En relación con la situación actual de las plazas de Mendoza, se observa que es una de las categorías más relevantes en la ciudad, por estar distribuida en la trama urbana de forma aleatoria conformando casi un $30 \%$ de los espacios analizados. Desde el punto de vista de la forma y la superficie, la plaza cuadrada con un área próxima a los $10.000 \mathrm{~m}^{2}$ es la tipología que más se repite en el ámbito en estudio, y es el resultado de la trama fundacional en damero con manzanas cuadradas de 100 por 100.

En cuanto al diseño adoptado, el modelo frecuente se relaciona con el tipo conmemorativo, en el cual se observa la presencia de grandes explanadas como sitio de reunión (se trata de un espacio abierto de materiales duros, expuesto a la radiación solar). Respecto de la organización interna mayoritaria, responde a una organización simétrica de los espacios, en torno de un núcleo central predominante sellado o semisellado, donde se encuentra un punto focal (fuente, monumento, etc.). La vegetación se sitúa en torno de ejes radiales que se originan desde el centro; estos son de relevancia paisajística, ya que priorizan la estética del espacio sin consideración en lo que respecta al uso de la vegetación para proporcionar sombra sobre los espacios de uso social. En cuanto al contexto de inserción, podemos ver que es variado y que estos espacios se insertan tanto en alta como en baja densidad edilicia, sin mostrar relación de dependencia con la zonificación de la ciudad de acuerdo con los usos del suelo.

Este análisis pone en evidencia la importancia de proponer diseños de áreas verdes eficientes que estén acordes con los condicionantes y limitaciones de cada ciudad, de modo de garantizar no solo la calidad ambiental y la habitabilidad del medio, sino también apuntar al bienestar social de sus habitantes. 


\section{Bibliografía}

ACERO, Juan y ARRIZABAGA, Jon (2010). "Influencia de la vegetación en la calidad del aire y el clima urbano". Congreso Nacional de Medio Ambiente, CONAMA 10, Madrid.

ARDDONE, Rita y BONNES, Mirilia (1996). "The urban green spaces in the psychological construction of the residential place”. En Bettini, V. (Ed.). Elementi di ecología urbana. Torino: Einaudi.

BORMIDA, Eliana (1984). "Mendoza, una ciudad oasis”. Revista de la Universidad de Mendoza, Facultad de Diseño, Arquitectura y Urbanismo. Mendoza, Argentina.

COLEY, Rebekah; KUO, Frances y SULLIVAN, William (1997). "Where does community grow? The social context created by nature in urban public housing”. Environment and Behavior, Vol. 29. Sage Publishing, North America.

CONWAY, Hazel (2000). "Parks and people: the social functions". The Regeneration of Public Parks. Taylor \& Francis, Reino Unido.

CORREA, Erica (2006). "Isla de Calor Urbana. El caso del área metropolitana de Mendoza”. Tesis doctoral. Universidad Nacional de Salta, Facultad de Ciencias Exactas, Salta, Argentina. DE ESTEBAN ALONSO, Alfonso (1982) Estudio comparado de estándares de equipamiento. Vol. 1. Teoría y Análisis. Ministerio de Obras Públicas y Urbanismo, Madrid.

DEL SAZ SALAZAR, Salvador y GARCÍA MÉNDEZ, Leandro (2007). "Estimating the nonmarket benefits of an urban park: Does proximity matter?”. Land Use Policy, Vol. 24 (1). Elsevier, Ámsterdam, Países Bajos.

HEISLER, Gordon (1986) “Energy savings with trees”. Journal of Arboricult, Vol. 12. USDA. Forest Service, Estados Unidos.

HEISLER, Gordon; GRIMMOND, Sue; GRANT, Rich y SOUCH, Catherine (1994) "Investigation of the influence of Chicago's urban forests on wind and air temperature.” General Technical Report. No. NE-186, US, Department of Agriculture, Forest Service, Northeastern. IRARRÁVAL, Felipe (2012). "El imaginario verde y el verde urbano como instrumento de consumo inmobiliario: configurando las condiciones ambientales del área metropolitana de Santiago”. INVI, Vol. 27 (75), Chile.

KAPLAN, Rachel (1993). "Urban forestry and the workplace”. En Gobster, P. H. (Ed.), Managing urban and high use recreation settings. General Technical Report NC-163. Forest Service, Chicago, USA.

KUO, Frances; BACAICOA, Magdalena y SULLIVAN, William (1998). "Transforming inner-city landscapes: Trees, sense of safety, and preference”. Environment and Behavior, Vol. 30, Sage Publishing, North America. 
Espacios verdes en ciudades de zona árida.

Diagnóstico de la situación actual de plazas de la ciudad de Mendoza, Argentina

LOIZU, Máximo (1980) Manual Municipal de Hacienda. CEUMT, Madrid.

LÓPEZ FALFÁN, Ina (2008). “Arbolado urbano Mérida Yucatán y su relación con aspectos socioeconómicos, culturales y de la estructura urbana de la ciudad”. Tesis de Maestría. Centro de Investigación y de Estudios Avanzados del Instituto Politécnico Nacional, Mérida, México.

MCPHERSON, Gregory (1988) "Functions of Buffer Plantings in Urban Environments". Agriculture, Ecosystems and Environment. Vol. 22/23, Netherlands.

(1994). "Energy-saving potential of trees in Chicago". Gen. Tech.

Rep. NE-186. Radnor, PA: USDA Forest Service, Northeastern Forest Experiment Station.

(1998). "Structure and sustainability of Sacramento's urban forest".

Journal of Arboriculture, Vol. 24 (4). Taylor \& Francis, on line, Reino Unido.

MCPHERSON, Gregory y SIMPSON, Jim (1995). "Shade trees as a demand - side resource". Home energy, Vol. 2. California, Estados Unidos.

MARTÍNEZ, Claudia; CANTÓN, Alicia y ROIG, Fidel (2009). "Impacto de la condición de aridez en el desarrollo ambientalmente sustentable de la ciudad oasis. El caso del arbolado urbano en el Área Metropolitana de Mendoza”. AVERMA, Vol. 13 (1), Argentina.

MORETTI, Graciela (2010). "Mendoza, la ciudad con dos centros históricos”. Ponencia presentada en el Seminario Centros Históricos y Centralidades Urbanas. Organizado por el Centro Internacional para la Conservación del Patrimonio (CICOP). Buenos Aires, Argentina.

MUNICIPALIDAD DE MENDOZA (2014) "Código urbano y de edificación 2000 - Ordenanza 3866/2014”. Mendoza, Argentina.

NIKOLOPOULOU, Marialena y STEEMERS, Koen (2003). “Thermal comfort and psychological adaptation as a guide for designing urban spaces”. Energy and Buildings, Vol. 35, Elsevier, Amsterdam, Países Bajos.

NOWAK, David (2002). "Understanding and Quantifying Urban Forest Structure, Functions and Value”. 5th Canadian Urban Forest Conference. October 2002. Region of York.

NOWAK, David y MCPHERSON, Gregory (1993) "Cuantificación del impacto ambiental de los árboles en Chicago. International Journal of Forestry and Forest Industries”. Unasylva, Vol. 173. FAO, Naciones Unidas.

PANASITI, Aída (1994) "Los espacios verdes en el ordenamiento territorial del Gran Mendoza”. Mendoza en el 2000. Proyecto de Ordenamiento Territorial para la Provincia. Plan Sectorial para el Gran Mendoza. Facultad de Ciencias Agrarias, Universidad Nacional de Cuyo, Argentina.

PRIEGO GONZALEZ DE CANALES, Carlos (2002). "Beneficios del arbolado urbano” 
Susana Stocco, María Alicia Cantón y Erica Correa

(ensayo de doctorado). Disponible en: http://digital.csic.es/bitstream/10261/24578/1/Beneficios\%20del\%20arbolado\%20urbano.pdf.

PONTE, Ricardo (2008) Mendoza, aquella ciudad de barro. Consejo Nacional Investigaciones Científicas Técnicas, CONICET. Mendoza, Argentina.

RIBAS I PIERA, Manuel (2002). "Paisaje y Ciudad”. Ciudades, N. 7, 2002-2003. Instituto Universitario de Urbanística de la Universidad de Valladolid, España.

SANTAMOURIS, Mattheos (2001) Energy and Climate in the Urban Built Environment. James and James Science Publishers, London.

SMITH, William (1990). Air Pollution and Forests. Springer-Verlag. New York.

SORENSEN, Mark; BARZETTI, Valerie; KEIPI, Kari y WILLIAMS, John (1998). "Manejo de las áreas verdes urbanas”. Documento de buenas prácticas (No. ENV-109). Banco Interamericano de Desarrollo, Washington, DC.

STOCCO, Susana; CANTÓN, Alicia y CORREA, Erica (2013). "Evaluación de las condiciones térmicas de verano y eficiencia ambiental de distintos diseños de plazas urbanas en Mendoza, Argentina”. Hábitat sustentable, Vol. 3, Chile.

(2015) "Design of urban green square in dry areas: Thermal performance and comfort”. Urban Forestry \& Urban Greening, N. ${ }^{\circ}$ 14, Elsevier, Amsterdam, Países Bajos.

ULRICH, Roger (1984). "View through a Window may Influence Recovery from Surgery". Science, Vol. 224, GALEGROUP Information Integrity. 